\title{
PREVENTABLE ERRORS: NEVER EVENTS
}

Narra Gopal Reddy ${ }^{1}$

\section{HOW TO CITE THIS ARTICLE:}

Narra Gopal Reddy. "Preventable Errors: Never Events". Journal of Evolution of Medical and Dental Sciences 2014; Vol. 3, Issue 29, July 21; Page: 8162-8172, DOI: 10.14260/jemds/2014/3027

ABSTRACT: Operation or any invasive procedure is a stressful event involving risks and complications. We should be able to offer a guarantee that the right procedure will be done on right person in the right place on their body. "Never events" are definable. These are the avoidable and preventable events. The people affected from consequences of surgical mistakes ranged from temporary injury in 60\%, permanent injury in 33\% and death in $7 \%$ ".World Health Organization (WHO) ${ }^{[1]}$ has earlier said that over seven million people across the globe suffer from preventable surgical injuries every year, a million of them even dying during or immediately after the surgery? The UN body quantified the number of surgeries taking place every year globally 234 million. It said surgeries had become common, with one in every 25 people undergoing it at any given time. $50 \%$ never events are preventable. Evidence suggests up to one in ten hospital admissions results in an adverse incident. This incident rate is not acceptable in other industries. In order to move towards a more acceptable level of safety, we need to understand how and why things go wrong and have to build a reliable system of working. With this system even though complete prevention may not be possible but we can reduce the error percentage ${ }^{2}$. To change present concept towards patient, first we have to change and replace the word patient with medical customer. Then our outlook also changes, we will be more careful towards our customers.

INTRODUCTION: Thousands of mistakes made in surgery and hundreds in anesthesia every year.[3] We probably think that surgical errors are more likely to occur during complex operations, such as brain, heart or spinal surgery. Actually, errors often occur in the most frequently performed operations.

Even after the main part of the surgery has been performed, medical negligence can happen in an instant. Any kind of major anesthesia or surgical errors can have disastrous results that cause long-lasting problems for the patient including death. These errors can be extremely devastating and cause serious health problems to our medical customers (patients). Unfortunately, many of these injuries might even cause long-lasting or permanent damage for a medical customer, leaving him or her not only with physical injury, but financial consequence as well.

Errors are common Complications commonly occur during surgery. But when you fall victim to such an occurrence, how do you know if it could have been avoided or why it even happened in the first place? Some complications are unpredictable and unpreventable. However, others stem from negligence, carelessness, poor planning, or distracted medical professionals. When you enter an operating room, patients (medical customers) have trust on us. Unfortunately, thousands of medical customers (patients) each year have their operations end in injury, disability, pain, emotional trauma, and sometimes death. 


\section{Most common medical mistakes made in the operating room [5];}

1. Anesthesia errors: Being "put under" with Anesthesia can be the most dangerous aspect of an operation. The anesthesiologist can fail to take proper medical history of the patient or neglect to inform of food, water and alcohol restrictions before surgery. During the surgery they may administer wrong amount of anesthetic, neglect to monitor the patient's vital parameters, or use defective medical devices.[5]

2. Damage resulting from poor surgical technique: If a surgeon uses tools carelessly, the results can be disastrous. A slip of scalpel, cattery, laser, or other surgical instrument can result in punctured or perforated membranes, severed nerves, and vital parts leads to internal bleeding, or infection. In some cases, negligent surgeon can leave behind tools or debris in the patient part, perform a wrong surgery, or even perform the surgery on the wrong part of the body or on a wrong patient.

3. Medication errors: According to a recent study, patients undergoing surgery are three times more likely to be harmed by medication errors than others in the health care system. ${ }^{[5]}$ Before, during and after surgery, many patients need a number of drugs, including antibiotics, sedatives and blood thinners etc. Healthcare professionals erroneously administer the wrong dose, wrong drug, at a wrong time. These mistakes can lead to over dosage, complication and even death.

4. Failure to react to complications properly: When a complication arises, the anesthetist or surgeon should react reasonably. However, if a healthcare professional fails to identify the issue, react in a timely manner or properly combat the problem, the result will be disastrous surgeons learn how to deal with complications such as hemorrhage and organ failure in the years of their training, so we should not accept any failure of action.

5. Unnecessary surgery: Healthcare professionals often pressurize patients into undergoing dangerous procedures that prove unnecessary. In fact, a research team recently revealed that up to 20percent surgeries could have been avoided.[5] Some of these unnecessary surgeries, such as coronary bypasses, hysterectomies, take place after doctors misinterpret symptoms or misread tests. Others take place simply because doctors want to make money. Either way, unnecessary surgery comes with a number of costs, including medical bills, pain and possible complications. ${ }^{[6]}$

CASE REPORTS: My Experience.

6. Wrong selection case: Gynecologist posted a case for laparoscopic hysterectomy. Patient was aged about 45years, obese, wt.80kgs, hypertensive, diabetic and with big fibroid uterus. It was her first case after training. She struggled for one hour without proceeding an inch further and with difficulty, open abdominal hysterectomy was done.

7. Restricted field x-ray: A case of accident sustained hip and knee injuries but x-ray was restricted to knee only later hip fracture is diagnosed - double operations - patient refused to pay second operation cost. 


\section{REVIEW ARTICLE}

8. Fast conclusions incomplete search: Double incision: For a case of Meckel's diverticulum perforation, incision taken for Appendectomy - normal appendix - signs of perforation - epigastria incision - normal findings -in-search perforated Meckel's diverticulum.

9. Laparotomy, Double Incisions in an Unmarried Girl: In a fresh Palsy case - pain abdomen diagnosed as perforated appendix - Mecburny incision - epigastic incision.

10. Wrong operation for a case of right lower lobe pneumonia: 15years old boy admitted with right side pain abdomen, posted for appendectomy, at laparotomy appendix found normal. On auscultation no breath sounds on lower part of right lung, x-ray revealed pneumonia right lower lobe.

The most common surgical error and the "never events" list for reference prepared by patient safety policy team 2012-13 of Great Britain?[7]:

1. Wrong site of surgery.

2. Wrong implant/prosthesis.

3. Retained foreign object after operation.

4. Wrongly prepared high-risk injectable medication.

5. Mal administration of potassium-containing solutions.

6. Wrong route administration of chemotherapy.

7. Wrong route administration of oral/enteral treatment.

8. Intravenous administration of epidural medication.

9. Maladministration of insulin.

10. Overdose of midazolam during conscious sedation.

11. Opioid overdose of an opioid-naive patient.

12. Inappropriate administration of daily oral drug.

13. Suicide using non-collapsible rails .

14. Escape of a transferred patient

15. Fall from unrestricted windows.

16. Entrapment in bedrails.

17. Transfusion of abo-incompatible blood components.

18. Transplantation of abo-incompatible organs as result of error.

19. Misplaced naso/ oro-gastric tubes.

20. Wrong gas administered.

21. Failure to monitor and respond to oxygen saturation.

22. Air embolism.

23. Misidentification of patients.

24. Severe scalding of patients.

25. Maternal death due to post-partum hemorrhage after elective caesarian section.

Number of Anesthesia Complications: The number of anesthesia complications from wrongful diagnosis was 0.724 per 1, 000 surgical discharges in the United States in 2000.[8] 


\section{The statistics also show:}

0.718 Per 1, 000 of complications in patients 0 -17age group.

0.580 Per 1,000 of complications in patients 18-44 age group

0.061 Per 1,000 of complications in patients 45-64age group.

0.833 Per 1,000 of complications in patients $>65$ age group.

\section{Causes of wrong-site surgeries system factors:[9]}

- Lack of institutional controls/formal system to verify the correct site of surgery.

- Lack of a checklist to make sure every check was performed.

- Exclusion of certain surgical team members.

- Reliance solely on the surgeon for determining the correct surgical site.

- Unusual time pressures (e.g., unplanned emergencies or large volume of procedures).

- Pressures to reduce preoperative preparation time.

- Procedures requiring unusual equipment or patient positioning.

- Team competency and credentials.

- Availability of information.

- Organizational culture.

- Orientation and training.

- Staffing.

- Environmental safety/security.

- Continuum of care.

- Patient characteristics, such as obesity or unusual anatomy that requires alterations in the usual positioning of the patient.

\section{Process factors:}

- Inadequate care planning.

- Inadequate medical record review.

- Miscommunication among members of the surgical team and the patient.

- More than one surgeon involved in the procedure.

- Multiple procedures on multiple parts of a patient performed during a single operation.

- Failure to include the patient and family or significant others when identifying the correct site.

- Failure to mark or clearly mark the correct operation site.

- Incomplete or inaccurate communication among members of the surgical team. Noncompliance with procedures.

- Failure to recheck patient information before starting the operation.

Examples of system errors defective/non-availability of healthcare equipments, instruments, disposables, drugs, supplies, etc:

1. Non-availability of the protocol/policy/plan (e.g., lack of infection control programs).

2. Inadequate training or supervision of doctors or other personnel.

3. Delay in provision or scheduling of services (e.g., lab tests, x-rays, follow-up visits). 


\section{REVIEW ARTICLE}

4. Inadequate reporting or communication (e.g., resident reporting late or not reporting at all due to failure of communication system).

5. Inadequate staffing involving various departments (e.g., during shift changes, weekends, holidays).

6. Inadequate functioning of hospital services (e.g., pharmacy, blood bank, housekeeping, etc.)

7. Lack of team work.

8. Inappropriate/inadequate discharge plan.

Commenting on the figures, Dr Mike Williams, a former hospital chief executive who lectures at Exeter University, said: 'I think it's just the tip of the iceberg.'

Hospital managers are now legally obliged to report all incidents leading to severe harm or death. "What is recorded is just a small amount of what happens".[10]

"There are mistakes in health care system that is not preventable. Infection rates will never get down to zero even if everyone does everything right. But the events we have estimated are totally preventable. For this we have to do a lot of careful work."

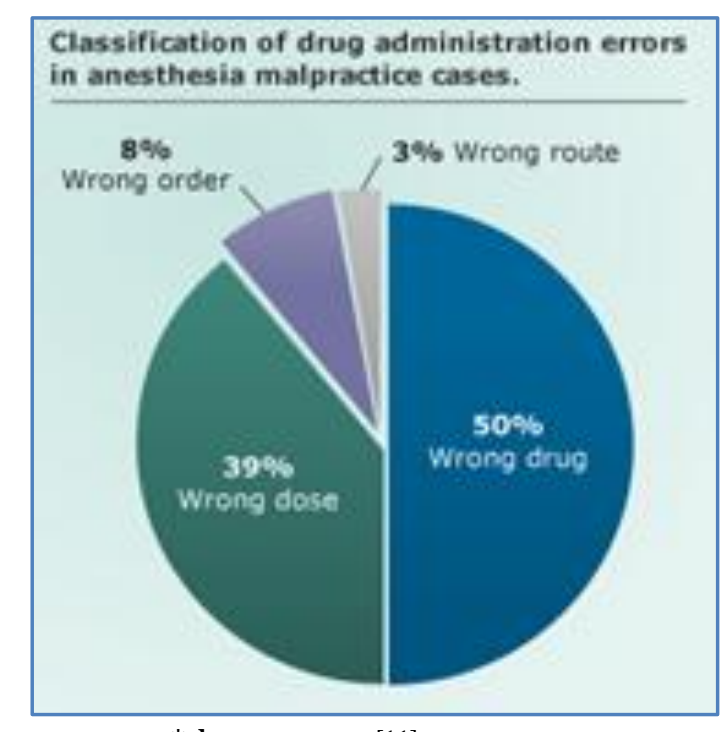

*drug errors[11]

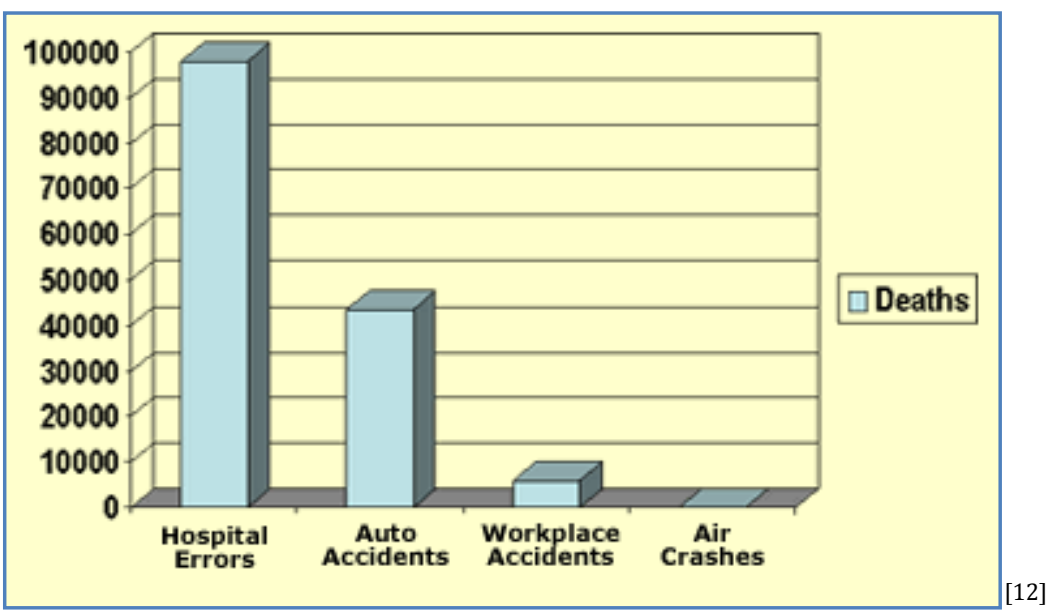




\section{Circumstances of injuries attributable to surgical errors, mention source of information phase} of care in which an error occurred:[13]

Phase of care in which error

Contributed to injury:

Preoperative management $39: 23 \%$

Intraoperative management $96: 68 \%$

Postoperative management 32:17

Severity of injury

Temporary disability $79: 54 \%$

Permanent disability $48: 33 \%$

Death 19: $13 \%$

Location of the adverse event

Operating room $\quad 8760 \%$

Hospital room (non-ICU) 23 16\%

Intensive care unit $\quad 1712 \%$

Physician's office $\quad 42 \%$

Emergency room $\quad 107 \%$

Other

$53 \%$

Medical Errors in India [4]: In India recording 5.2 million injuries every year due to medical errors adverse events. Topping the list is:
a. Medication errors
b. Hospital acquired infections
c. Deep vein thrombosis.

Due these injuries 3 million yeas healthy lives lost. Worldwide recording 43 million injuries, 23 million year yeas healthy lives lost.

Doctor from HARVARD school of public health has concluded that more than 43 million people are these injuries result in the loss of nearly 23 million years of healthy life.

- These findings for the first time try to quantify the global burden of unsafe medical care across a range of adverse health events.

"This is the first attempt to quantify the human suffering those results from unsafe care," said lead author ashish jha, professor of health policy and management at HSPH. "We find that millions of people around the world are hurt, disabled and sometimes even die as a result of medical errors".

*Data source WHO[14]

\begin{tabular}{|c|l|c|c|c|c|c|c|}
\hline $\begin{array}{c}\text { Sl. } \\
\text { no. }\end{array}$ & \multicolumn{1}{|c|}{$\begin{array}{c}\text { Surgical errors/ } \\
\text { complications }\end{array}$} & $\begin{array}{c}\text { No. Of } \\
\text { cases }\end{array}$ & $\begin{array}{c}\text { \% } \\
\text { per1000 }\end{array}$ & $\begin{array}{c}\text { Survival } \\
\text { rate }\end{array}$ & Deaths & \% per 1000 & $\begin{array}{c}\text { extra } \\
\text { cost }\end{array}$ \\
\hline 1 & $\begin{array}{l}\text { Accidental puncture/ } \\
\text { laceration }\end{array}$ & 111989 & 3.09 & 92.35 & & 7.65 & 463.13 \\
\hline 2 & Foreign body left & 2591 & 0.07 & 93.75 & 55 & 6.25 & 17.25 \\
\hline 3 & $\begin{array}{l}\text { Anesthesia } \\
\text { complications/ } \\
\text { survival \% }\end{array}$ & 2357 & 0.24 & 98.56 & 726 & 1.44 & 1.88 \\
\hline 4 & $\begin{array}{l}\text { Haemorrhage/ } \\
\text { haematoma }\end{array}$ & 24108 & 2.46 & 89.55 & & 10.45 & 258.33 \\
\hline
\end{tabular}


REVIEW ARTICLE

\begin{tabular}{|c|c|c|c|c|c|c|c|}
\hline 5 & $\begin{array}{l}\text { Physiological/ } \\
\text { metabolic }\end{array}$ & 6700 & 1.35 & 76.88 & 1327 & 23.12 & 183.64 \\
\hline 6 & $\begin{array}{l}\text { Post -op pulmonary } \\
\text { embolism }\end{array}$ & 128738 & 8 & 93.44 & 8445 & 6.56 & 1397.39 \\
\hline 7 & $\begin{array}{l}\text { Post-op respiratory } \\
\text { failure }\end{array}$ & 28940 & 8.1 & 69.03 & 6320 & 30.97 & 774.17 \\
\hline 8 & Wound dehiscence & 6384 & 3.76 & 85.01 & 615 & 14.99 & 128.71 \\
\hline 9 & Infections & 88286 & & 86.84 & 3805 & 13.16 & 1706.39 \\
\hline 10 & Transfusion reactions & 190 & 0.01 & 91.05 & 17 & 8.95 & 33.67 \\
\hline 11 & Un diagnosed & 187289 & & 95.69 & 24 & 4.31 & 1397.39 \\
\hline 12 & Total & 587572 & 27.08 & 972.15 & 21334 & 127.85 & 6361.95 \\
\hline
\end{tabular}

- data of malpractice claim information to surgical never events from the national practitioner data bank from 1990 to 2010 of USA[4]

\begin{tabular}{|c|l|c|c|c|c|}
\hline $\begin{array}{c}\text { Sl. } \\
\text { No. }\end{array}$ & \multicolumn{1}{|c|}{ Events } & $\begin{array}{c}\text { Developed } \\
\text { countries }\end{array}$ & $\begin{array}{c}\text { Developing } \\
\text { countries }\end{array}$ & Total & India \\
\hline 1 & Surgeries & 80 millions & 154 millions & $\begin{array}{c}234 \\
\text { millions }\end{array}$ & 29 millions \\
\hline 2 & Errors & 14 million & 27 millions & 43 millions & 5.2 millions \\
\hline 3 & Readmissions & $14.20 \%$ & $12.70 \%$ & $13.50 \%$ & $12.50 \%$ \\
\hline 4 & Drug errors & $5 \%$ & $9 \%$ & $6.50 \%$ & $9.50 \%$ \\
\hline 5 & Infections & $2.50 \%$ & $3 \%$ & $2.90 \%$ & $4 \%$ \\
\hline 6 & $\begin{array}{l}\text { Temporary } \\
\text { disability }\end{array}$ & $59 \%$ & $55 \%$ & $56 \%$ & $55 \%$ \\
\hline 7 & $\begin{array}{l}\text { Permanent } \\
\text { disability }\end{array}$ & $33 \%$ & $35 \%$ & $33.60 \%$ & $35 \%$ \\
\hline 8 & $\begin{array}{l}\text { Deaths (major } \\
\text { surgeries) }\end{array}$ & $6.60 \%$ & $10 \%$ & $8 \%$ & $! 0 \%$ \\
\hline 9 & Working days lost & 7.2 million days & 15.5 million/d & $\begin{array}{c}22.7 \\
\text { million } / \mathrm{d}\end{array}$ & $3.5 \mathrm{million} / \mathrm{d}$ \\
\hline 10 & $\begin{array}{l}\text { Financial } \\
\text { implications }\end{array}$ & $\$ 1.3$ billion & $\$ 1.5$ billion & $\$ 2.8$ billion & $\$ 0.4$ billion \\
\hline
\end{tabular}




\section{REVIEW ARTICLE}

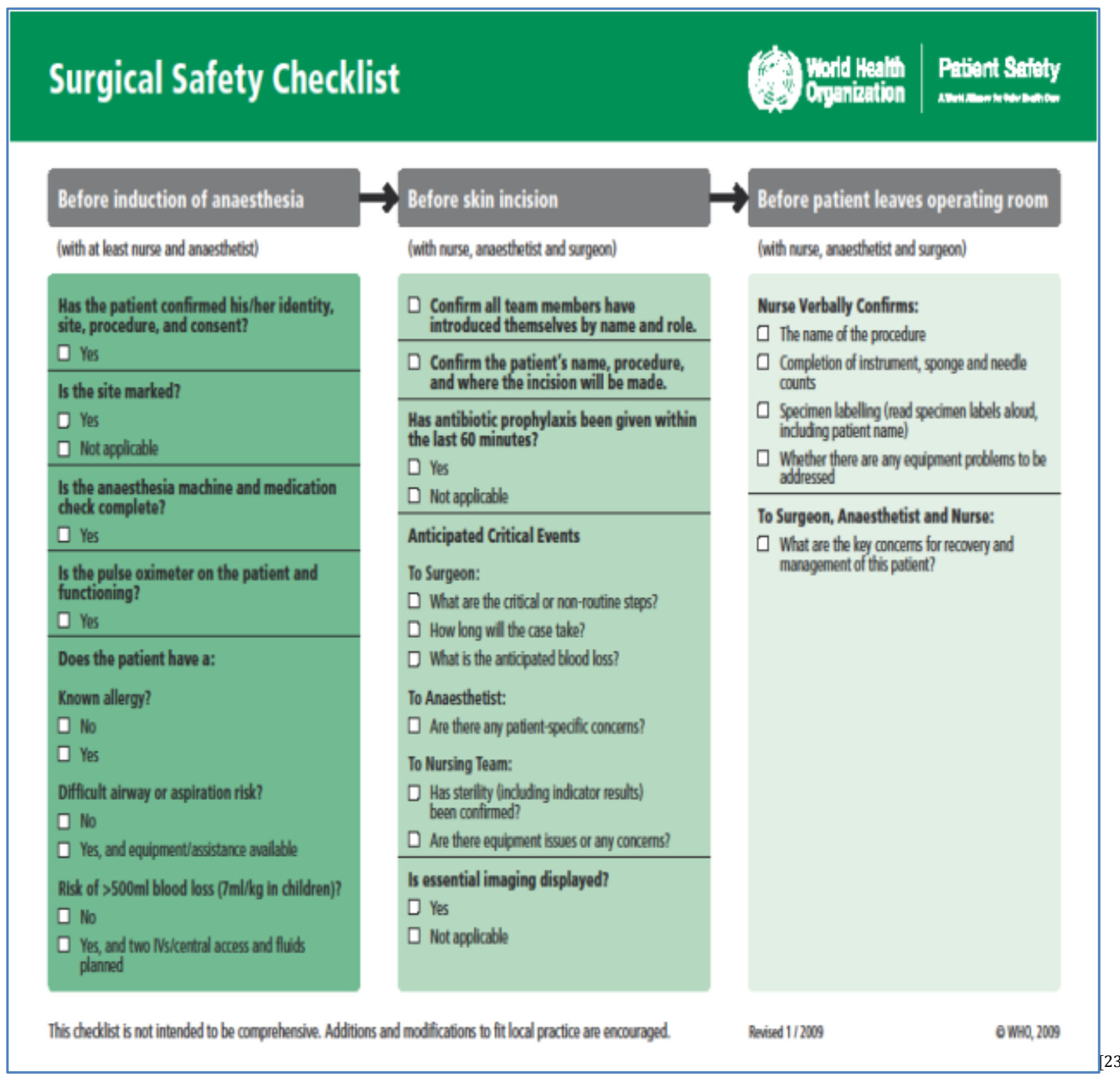

[23]

DISCUSSION: Speaking to TOY, Dr Jha said "unfortunately, our data from India is among the poorest there have been very few studies done on this topic and so we are making estimations based on a global average for low and middle income countries, with only a few data inputs from India itself

Who has earlier said that over seven million people across the globe suffer from preventable surgical injuries every year, a million of them even dying during or immediately after the surgery? Quote.[2]

The UN body quantified the number of surgeries taking place every year globally 234 million. It said surgeries had become common, with one in every 25 people undergoing it at any given time. China conducted the highest number of surgeries followed by Russia and India. ${ }^{[4]}$

"When we report in the low end of the range because many never events go unreported" says researcher Marty Makary MD, mph. Associated professor of surgery at Johns Hopkins university 
school of medicine. Makary says by law, hospitals are required to report never events that result in a settlement of judgment. But not all items left behind after surgery are discovered.

They are typically only reported when a patient experiences a complication after surgery and doctors try to find out why. "We believe the events we describe are real" says Maker "I cannot imagine a hospital paying out a settlement for a false claim or a retained sponge" the consequences of surgical mistakes ranged from temporary injury in 59\% of the cases to death in $6.6 \%$ of the cases and permanent injury in $33 \%$ of people affected.

In this document exploring how approaches such as human factors can help us to make 'never' a near reality. Here patients, their families, clinicians and their teams, all who thought it would never, could never happen to them. We will then look at how we can learn from these cases to ensure that next time it doesn't happen to us. Researchers say most medical centers have long list of patient safety procedures to place to prevent surgical mistakes such as mandatory "time-outs" in the operating room to make sure medical records and surgical plans match the patient on the table. Even though these errors are not intentional but require more vigilance, competence and care from medical industry.[15-22,24]

CONCLUSION: Researchers say most medical centers have long patient safety procedures in place to prevent surgical mistakes as:

1. Following peri-operative protocols like, proper history, system wise examination, necessary investigations, correction of hypertension, diabetes, hob, and other parameters to near normal, patient preparation, consent protocols, aseptic precautions and anti-biotic protocols.

2. Following universal protocol like "sign in" "time out" and "sign out" at time surgery. Postoperative, discharge protocols.

3. New technology use, like surgical sponges with radio frequency tags that can be detected by a scanner, electronic tagging for drugs. Electronic recording of operation theatre proceedings and print copy of vital data.

4. In addition to adopting new technologies to increase patient safety, Makary says a new standardized system of reporting surgical mistakes is needed to properly measure and address the problem finally. Makary and fry say greater attention should be paid to the culture of safety and team work among nurses, surgeons, and everyone else in the operating room. For known sources of risk there are existing guidelines and safety recommendations which are to be followed to prevent the event. There is worldwide support for implementation of preventable guidance.

5. Good training, knowledgeable care, competence, dedication to ward profession, customer care is key for achieving goals.

6. Finally to prevent this present concept of word patient has to be changed and to be replaced with word medical customer. Then our outlook also changes, we will be more careful towards our customers. 


\section{REFERENCES:}

1. Medical-dictionary.thefreedictionary.com/never event Cached Segen's Medical Dictionary. (C) 2012 Farlex, Inc. All rights reserved.

2. Timesofindia.indiatimes.com/india/Millions-die-of. 'Millions die of preventable surgical injuries. Seven million people across the globe suffer from preventable surgical injuries every year, a million.

3. Laura Landro , "Surgeons Make Thousands of Snafus", The Wall Street Journal: A2, retrieved 12 October 2013, (subscription required (help).

4. 'India records 5.2 million medical injuries a year' The author has posted comments on this article kounteya Sinha, TNN | Sep 21, 2013, 05.55AM IST.

5. Www.siegelandcoonerty.com/library/common-medical-errors Cach Five common medical mistakes of Newark surgeons.

6. www.usatoday.com/story/news/nation/2013/06/18/ Cached doctors perform thousands of unnecessary surgeries. Tens of thousands of times each year, patients undergo surgery that isn't necessary.

7. Www.gov.uk/government/uploads/system/uploads/. The "Never Events" list has been updated with amendments to two of the never event definitions. The changes are to never events 18, ABO-... Department of Health.

8. Www.ncbi.nlm.nih.gov/books/NBK2678 a wrong site surgery preventable error. 8(a) Incidents of statistics of about anaesthesia complications - Right diagnosis http://www.rightdiagnosis.com/a/anesthesia_complications/stats.htm

9. www. Causes of Wrong-Site Surgeries

10. www.dailymail.co.uk/news/article-2496114,www.ukmalayalee.com/ukews/news.php?Id=mza2oa== Cached Errors in acute hospital. That was largely due to new reporting rules stating all... Hospital managers are now legally obliged to report all incidents.

11. Classification of drug administration errors in anesthesia malpractice. More Webmm.ahrq.gov

12. www.emsa.ca.gov/Media/Default/Word/AMTF_paper_final.doc The Federal Aviation Administration rather than to the base hospital.

13. Atul A. Gawande, MD, MPH, Michael J. Zinner, MD, David M. Studdert, LLB, scd, MPH, Troyen A. Brennan, MD, JD, MPH . Analysis of errors reported by surgeons at three teaching hospitals. Surgery 2003;133:614-21.

14. Michaels, Robert K. MD, MPH; Makary, Martin A. MD, MPH†; Dahab, Yasser MD*; Frassica, Frank J. MD; Heitmiller, Eugenie MD; Rowen, Lisa C. RN, dnsc§; Crotreau, Richard MD; Brem, Henry MD; Pronovost. Achieving the National Quality Forum's "Never Events": Prevention of Wrong Site, Wrong Procedure, and Wrong Patient Operations. Ann surgery. April2007,24(4) 526-532

15. Gawande aa, zinner mj, studdert dm, brennan ta. Analysis of errors reported by surgeons at three teaching hospitals. Surgery 2003; 133: 614-21.

16. Joint commission on accreditation of health care organizations. Root cause analysis in health care: tools and techniques. Oakbrook terrace (il): joint commission on accreditation of health care organizations; 2000.

17. Cheney fw. The american society of anesthesiologists closed claims project: what have we learned, how has it Affected practice, and how will it affect practice in the future? Anesthesiology 1999; 91(2): 552-66. 
18. Eichhorn jh, cooper jb, cullen dj, maier wr, philip jh, seeman rg. Standards for patient monitoring during Anesthesia at harvard medical school. Jama 1986; 256: 1017-20.

19. www.oshmanlaw.com/Harvard-Medical-Practice-Study.pdf...results of the Harvard Medical Practice Study Investigators. Study of medical injury and malpractice. Patients, doctors and lawyers: Qual Saf Health Care 2004;13:151-152 doi:10.1136/qshc.2002.003905

20. Clinical human factors group final version February 2012. Clinical human factors group final version February 2012. $7^{\text {th }}$ open seminar, Loughborough;2014 25th June

21. Jennifer warner. webmed health news reviewed by laura j. Martin, Department of health, 2011, the 'never events' list for 2011/12: policy framework for use in the Nhs. London: $\mathrm{dh}$.

22. Flin, r., o'connor, $\mathrm{p}$ and crichton, $\mathrm{m}$. safety at the sharp end: a guide to non-technical Skills. Aldershot: ashgate. References(2008).

23. Department of health, 2011, the 'never events' list for 2011/12: policy framework for use in the Nhs. London: dh.

24. standards and recommendations for safe perioperative practice, 2007, available at http://www.afpp.org.uk/news/safe-practice-highlighted-in-new-afpp-publication - swab, instrument and needle counts: managing the risk, 2005, available at http://www.afpp.org.uk/filegrab/swabcountposter.pdf?ref=57 - patient safety alert - who surgical safety checklist, 2009, available at http://www.nrls.npsa.nhs.uk/resources/clinicalspecialty/surgery/.

\section{AUTHORS:}

1. Narra Gopal Reddy

\section{PARTICULARS OF CONTRIBUTORS:}

1. Associate Professor, Department of Anaesthesiology, KIMS, NKP.

\section{NAME ADDRESS EMAIL ID OF THE} CORRESPONDING AUTHOR:

Dr. N. Gopal Reddy, Plat No. 54, Old V. T. Colony, Nalgonda-508001, A. P.

Email: drgopalreddynarra@yahoo.com

Date of Submission: 16/06/2014.

Date of Peer Review: 17/06/2014.

Date of Acceptance: 26/06/2014.

Date of Publishing: 19/07/2014. 